treatments help symptoms although they do not treat the underlying cause.

Even if we do elicit our patients' explanatory model, how much will it change the treatment we give them? For instance, consider an African patient who, in terms of an ICD-10 diagnosis, is suffering from a hypomanic episode. $\mathrm{He}$ is physically violent. Both he and his family hold that he is possessed by a spirit. Are we to accept their explanatory model and enlist an exorcist? Will we withhold pharmacological treatment because the patient holds an alternative view of his illness?

What is needed is an approach in transcultural psychiatry that looks at not just what people believe but what they actually do in practice. A comprehensive approach involving participant observation, not just the administration of questionnaires to patients, will lead to greater understanding.

Bhui, K. \& Bhugra, D. (2002) Explanatory models for mental distress: indications for clinical practice and research. British Journal of Psychiatry, 18I, 6-7.

Callan, A. \& Littlewood, R. (1998) Patient satisfaction: ethnic origin or explanatory model? International Journal of Social Psychiatry, 44, I-II.

Dein, S. (200I) The use of traditional healing in South Asian psychiatric patients in the UK: interactions between professional and folk psychiatrists. Transcultural Psychiatry, 38, 245-259.

Kleinman, A. (1980) Patients and Healers in the Context of Culture. Berkley, CA: University of California Press.

Last, M. (1981) The importance of knowing about not knowing. Social Science and Medicine, I5, 387-392.

Pelto, P. J. \& Pelto, G. H. (1997) Studying knowledge, culture and behaviour in applied medical anthropology. Medical Anthropology Quarterly, II, 147-163.

Weiss, M. G., Doongaji, D. R., Siddhartha, S., et al (1992) The Explanatory Model Interview Catalogue (EMIC). Contributions to cross-cultural research methods from a study of leprosy and mental health. British Journal of Psychiatry, 160, 819-830.

Williams, B. \& Healy, D. (200I) Perceptions of illness causation among new referrals to a community health team: explanatory model or exploratory map? Social Science and Medicine, 53, 465-476.

S. Dein Princess Alexandra Hospital, The Derwent Centre, Hamstel Road, Harlow, Essex CM20 IQX, UK

\section{Pathways to care in ADHD}

I was interested to read Sayal et al's (2002) article on pathways to care for children at risk of attention-deficit hyperactivity disorder (ADHD). By using Goldberg \& Huxley's (1980) pathway to care model I felt that the study oversimplified the complexity of professional input to this group of children, a point raised by the authors in their discussion. I think it is important, when considering improvements to services for children with ADHD, that the role of education is highlighted.

Teachers, as a profession, are well placed to observe children and are familiar with age-appropriate behaviour. Indeed, Goodman et al (2000) found that teachers were more sensitive at identifying children with hyperactivity than were their parents. Although teachers' involvement in the assessment and monitoring of children with ADHD is well established (Dulcan et al, 1997), their role in identification is less clear. This is highlighted by the fact that only some child and adolescent mental health services (CAMHS) accept referrals directly from schools. By involving teachers in the identification of children with ADHD, access to children would improve from $74 \%$ seen in primary care to nearly $100 \%$. This would significantly improve the sensitivity of any screening measure.

It is essential that CAMHS do not develop services for children in isolation, but instead utilise the skills of other professionals to improve care. If children with ADHD are to have their needs met, it is essential that we start to think outside of the medical model.

Dulcan, M. and the Work Group on Quality Issues, American Academy of Child and Adolescent Psychiatry (1997) Practice parameters for the assessment and treatment of children, adolescents and adults with attention-deficit/hyperactivity disorder. Journal of American Academy of Child and Adolescent Psychiatry, 36 (suppl.), 855-1215.

Goldberg, D. \& Huxley, P. (1980) Mental Illness in the Community: The Pathway to Psychiatric Care. London: Tavistock.

Goodman, R., Ford, T., Simmons, H., et al (2000) Using the Strengths and Difficulties Questionnaire (SDQ) to screen for psychiatric disorders in a community sample. British Journal of Psychiatry, 177 534-539.

Sayal, K., Taylor, E., Beecham, J., et al (2002) Pathways to care in children at risk of attention-deficit hyperactivity disorder. British Journal of Psychiatry, $\mathbf{1 8} \mathbf{I}$, $43-48$.

J. Cribb Young People's Service, 3 North Parade Buildings, Bath BAI INS, UK

Authors' reply: Dr Cribb's comments are welcomed. Our discussion (Sayal et al, 2002) also highlights the potential role of both parents and teachers in making referrals to child and adolescent mental health services (CAMHS). The paper is developing new methodology and we deliberately posed it on a simplified system, selecting an area where most referrals come from general practitioners (GPs). GPs are also the main referrers to CAMHS nationally and their role in primary care trusts will be of great importance in shaping specialist services. Nevertheless, this is only one component of tier 1 services and $48 \%$ of CAMHS referrals come from other sources (Audit Commission, 1999). We plan to widen our programme to examine the role of other sources of referral.

Restricting referrals to particular agencies imposes barriers to access, and the resulting delay in referrals might exacerbate severity or chronicity of problems. Kurtz et al (1996) described a service that only accepted GP referrals. It failed to reduce the number of referrals and generated resentment from other agencies. Comparisons of CAMHS with different referral systems will improve knowledge in quantifying the barriers to access to services. This could contribute to assisting the successful implementation of the National Service Framework for Children.

The role of teachers in the pathway to care merits particular comment. Relationship difficulties with teachers are a predictor of referral of hyperactive children to CAMHS (Woodward et al, 1997). Our study has demonstrated that selective targeting can lead to particularly high rates $(98 \%)$ of teacher participation in research. This is likely to reflect their concern about behavioural and emotional difficulties in children. Teachers are a rich potential source of child mental health information for parents. However, in considering referrals from schools, it is imperative that teachers fully discuss their concerns with parents. Parents need to agree to any referral. For hyperactivity, in particular, it needs to be ascertained that the problems are pervasive. Unless this happens, there is a risk that learning difficulties are wrongly identified as hyperactivity. This also highlights the importance of adequately resourced educational psychology services to support schools, and health service input in the training of teachers.

Audit Commission (1999) Children in Mind. Child and Adolescent Mental Health Services. London: Audit Commission.

Kurtz, Z., Thornes, R. \& Wolkind, S. (1996) Services for the Mental Health of Children and Young People in England: Assessment of Needs and Unmet Need. Report to the Department of Health. London: South Thames Regional Health Authority. 
Sayal, K., Taylor, E., Beecham, J., et al (2002) Pathways to care in children at risk of attention-deficit hyperactivity disorder. British Journal of Psychiatry, I8I, 43-48.

Woodward, L., Dowdney, L. \& Taylor, E. (1997) Child and family factors influencing the clinical referral of children with hyperactivity: a research note. Journal of Child Psychology and Psychiatry, 38, 479-485.

\section{K. Sayal, E. Taylor, J. Beecham, P. Byrne}

Institute of Psychiatry, De Crespigny Park, Denmark Hill, London SE5 8AF, UK

\section{Consciousness still a mystery}

Baroness Greenfield's (2002) editorial is shaped by the metaphors of empiricism. The brain is a network. The mind is distinct patterns of neural connectivity. Currently, the evidence for such a scenario is limited. Brain connections may, as she says, 'actually reflect experience', but no pattern of connectivity has ever been related to any particular mental state. The alternative hypothesis of functional specialisation merits more than the scant consideration granted in the editorial, given the recent interest in the notion of modularity (Fodor, 1983; Pinker, 1999). Perversely, Greenfield chooses to support her 'network' hypothesis by reference to a study showing regionally localised brain changes in taxi drivers (Maguire et al, 2000).

Consciousness is introduced as a dimensional variable quantifying the current extent of this connectivity. Seemingly, the more connected our brains are the more conscious we are. But is this anything more than metaphorical fooling around? She presents no evidence for what a conscious brain state might look like. Where consciousness occurs is surely rather an unimportant issue. The hard question, which
Greenfield ignores, is 'How can pain (which hurts so) possibly be the same thing as insensate molecules rushing around in nerve fibres?' (Papineau, 2002). On this our ignorance remains as complete as it ever was.

Fodor, J. A. (1983) The Modularity of Mind. Cambridge, MA: MIT Press.

Greenfield, S. (2002) Mind, brain and consciousness. British Journal of Psychiatry, 18I, 91-93.

Maguire, E. A., Gadian, D. G., Johnsrude, I. S., et al (2000) Navigation related structural change in the hippocampi of taxi drivers. Proceedings of the National Academy of Sciences of the USA, 97, 4398-4403.

Papineau, D. (2002) Thinking About Consciousness. Oxford: Oxford University Press.

Pinker, S. (1999) How the Mind Works. London: Penguin.

H. Jones Maudsley Hospital, Denmark Hill, London SE5 8AF, UK

\section{One hundred years ago}

\section{Ladislav Haskovec and akathisia: 100th anniversary}

Akathisia is a syndrome of objective and subjective motor restlessness manifested by an inability to sit or stand still. The patients are distressed and they pace constantly. Today, it is mostly known as a side-effect of antipsychotic medications. However, the phenomenon was observed before the introduction of antipsychotics, and the term 'akathisia' (derived from the Greek 'inability to sit') was coined in 1901 by Ladislav Haskovec, MD. A Czech neuropsychiatrist, Haskovec was born in 1866 and died in 1944.

After graduation from the Charles University School of Medicine in Prague, he spent a year in Paris working with Professor Charcot, the leading neurologist at that time. His original primary interest was neuropathology, but he soon branched out into many other areas. He published on thyroid function, tuberculosis, alcoholism, neuroses, obsessions, mechanisms of consciousness, seizure disorders and heredity. His publications and presentations earned him international recognition and numerous honours in Austria, Czechoslovakia and France. He was appointed full professor at the Charles University in 1919, and served as Dean of the Charles University School of Medicine in 1925-1926.
Throughout his long career, Haskovec was an astute clinician. He coined the term 'akathisia' to describe symptoms he observed in two of his patients. These two case reports were presented at the meeting of the Sociéte de Neurologie in Paris on 7 November 1901 (Haskovec, 1901). (English translations of Haskovec's papers with a commentary were published elsewhere (Berrios, 1995).) The patients were adult males who had a multitude of symptoms including insomnia, vertigo, various aches and pains, and paraesthesias. Both men complained of generalised tremor; apparently this was not observed during examination. The prominent symptom in both patients was that they were unable to remain sitting down for any length of time. When sitting, at least one of the patients had a sensation in his legs as if he were jumping (today, a clinician would perhaps describe this sensation as a feeling of restlessness). The movements were described as involuntary by the patients who actually wanted to stop them; one of them tried to hang on to a table to prevent himself from getting up. After jumping up from the sitting position, the patient kept walking around, and conversation with him was only possible when he was moving. Gait was normal in both patients; neurological examination revealed no clear abnormalities, and there were no signs of psychosis.
Haskovec tentatively diagnosed one man with 'hysteria' and the other one with 'neurasthenia'. He speculated about underlying mechanisms along the lines of 'hyperexcitability' or 'fatigue' of various brain structures, using theoretical concepts of his era.

The report elicited discussions with French neurologists who accepted the new term but wanted to apply it differently from its originator (Haskovec, 1903). Nevertheless, today's phenomenology of akathisia remains essentially the same as described by Haskovec 100 years ago. Neurology textbooks of the pre-antipsychotic era described akathisia in Parkinsonian patients, and the importance of the term has further increased after the introduction of antipsychotics in the 1950s. Neurologists and psychiatrists are indebted to Haskovec for his astute observations.

Berrios, G. E. (1995) Lad Haskovec and akathisia: an introduction. History of Psychiatry, 6, 243-25I.

Haskovec, L. (190I) L'akathisie. Revue Neurologique, 9 |107-1109.

- (1903) Nouvelles remarques sur l'akathisie. Nouvelle Iconographie de la Salpêtrière, I6, 287-296.

Pavel Mohr Prague Psychiatric Center, Charles University of Prague, Ustavni 9I, I8I 03 Praha 8, Czech Republic

Jan Volavka Nathan Kline Institute, Orangeburg, New York University, New York, USA 